\title{
Pest control of aphids depends on landscape complexity and natural enemy interactions
}

Emily A. Martin, Björn Reineking, Bumsuk Seo, Ingolf Steffan-Dewenter

Aphids are a major concern in agricultural crops worldwide, and control by natural enemies is an essential component of the ecological intensification of agriculture. Although the complexity of agricultural landscapes is known to influence natural enemies of pests, few studies have measured the degree of pest control by different enemy guilds across gradients in landscape complexity. Here, we use multiple natural-enemy exclosures replicated in 18 fields across a gradient in landscape complexity to investigate 1) the strength of natural pest control across landscapes, measured as the difference between pest pressure in the presence and in the absence of natural enemies; 2 ) the differential contributions of natural enemy guilds to pest control, and the nature of their interactions across landscapes. We show that natural pest control of aphids increased up to six-fold from simple to complex landscapes. In the absence of pest control, aphid population growth was higher in complex than simple landscapes, but was reduced by natural enemies to similar growth rates across all landscapes. The effects of enemy guilds were landscape-dependent. Particularly in complex landscapes, total pest control was supplied by the combined contribution of flying insects and ground-dwellers. Birds had little overall impact on aphid control. Despite evidence for intraguild predation of flying insects by ground-dwellers and birds, the overall effect of enemy guilds on aphid control was complementary. Understanding pest control services at large spatial scales is critical to increase the success of ecological intensification schemes. Our results suggest that, where aphids are the main pest of concern, interactions between natural enemies are largely complementary and lead to a strongly positive effect of landscape complexity on pest control. Increasing the availability of seminatural habitats in agricultural landscapes may thus benefit not only natural enemies, but also the effectiveness of aphid natural pest control. 


\section{Pest control of aphids depends on landscape complexity and natural}

2

3

4

5

6

7

8

9 Hubland, 97074 Würzburg, Germany.

10 bBiogeographical Modelling, Bayreuth Center for Ecology and Environmental Research

11 BayCEER, University of Bayreuth, Universitätstraße 30, 95447 Bayreuth, Germany.

12 cUR EMGR Écosystèmes Montagnards, Irstea, 2 rue de la Papeterie-BP 76, F-38402, St-Martin-

13 d'Hères, France

14 dDepartment of Plant Ecology, University of Bayreuth, Universitätstraße 30, 95447 Bayreuth,

15 Germany.

16 *Corresponding author: emily.martin@uni-wuerzburg.de.

17

18

19

20

21

22

\section{enemy interactions}




\section{Summary}

24 Aphids are a major concern in agricultural crops worldwide, and control by natural enemies is an essential component of the ecological intensification of agriculture. Although the complexity of agricultural landscapes is known to influence natural enemies of pests, few studies have measured the degree of pest control by different enemy guilds across gradients in landscape complexity. Here, we use multiple natural-enemy exclosures replicated in 18 fields across a gradient in landscape complexity to investigate 1) the strength of natural pest control across landscapes, measured as the difference between pest pressure in the presence and in the absence of natural enemies; 2) the differential contributions of natural enemy guilds to pest control, and the nature of their interactions across landscapes. We show that natural pest control of aphids increased up to six-fold from simple to complex landscapes. In the absence of pest control, aphid 34 population growth was higher in complex than simple landscapes, but was reduced by natural enemies to similar growth rates across all landscapes. The effects of enemy guilds were landscape-dependent. Particularly in complex landscapes, total pest control was supplied by the combined contribution of flying insects and ground-dwellers. Birds had little overall impact on aphid control. Despite evidence for intraguild predation of flying insects by ground-dwellers and birds, the overall effect of enemy guilds on aphid control was complementary. Understanding pest control services at large spatial scales is critical to increase the success of ecological intensification schemes. Our results suggest that, where aphids are the main pest of concern, interactions between natural enemies are largely complementary and lead to a strongly positive effect of landscape complexity on pest control. Increasing the availability of seminatural habitats in agricultural landscapes may thus benefit not only natural enemies, but also the effectiveness of aphid natural pest control. 
Introduction

Pest control by natural enemies is an essential ecosystem service valued at $\$ 4.49$ billion annually in the USA alone (Losey and Vaughan 2006). In the context of increasing pressure to reduce harmful chemical inputs while maintaining or increasing current agricultural yields, the gradual replacement of conventional agricultural practices with natural pest control provided by functional ecosystems is a major hope for the future (Bommarco et al. 2013). However, current understanding of the flow and stability of pest control services in human-modified systems is limited, and constrains our ability to implement an ecological intensification of agriculture (Rusch et al. 2013). to be influenced both by local management intensity (Bengtsson et al. 2005) and by the landscape context surrounding crop fields (Bianchi et al. 2006; Rand et al. 2012). In landscapes with high amounts of complex or seminatural habitats, enemies such as parasitoid wasps, predatory beetles, and insectivorous birds are frequently more abundant and species-rich than in

61 simplified landscapes with large monocultures and little complex habitat. These effects vary 62 according to the enemies considered and the spatial scale of observations (Chaplin-Kramer et al. 63 2011), and are strongest for enemies that depend on permanent habitat structures for nesting, 64 overwintering and other food resources (Bianchi et al. 2006). Within crop fields, these landscape 65 effects can be intensified by spillover, defined as the flow of organisms occurring across the 66 interface between crop and non-crop habitats (Tscharntke et al. 2005). In contrast, effects of

67 landscape context on pest distributions are less well understood and appear highly variable 68 between systems and years (Bianchi et al. 2006; Chaplin-Kramer et al. 2011; O'Rourke et al. 
69 2011; but see Meehan et al. 2011). As pest distributions are a reflection of landscape effects on

70 the pests themselves, but also of their suppression by natural enemies in the same landscape over

71 more than one season (O'Rourke et al. 2011; Chaplin-Kramer and Kremen 2012), estimating the

72 effects of landscape context on pests and thus on pest suppression requires distinguishing pest

73 abundance in the presence, vs. in the absence of enemies. However, the direct effect of landscape

74 context on the strength of pest suppression by natural enemies has only rarely been measured

75 (Chaplin-Kramer et al. 2011) and may not show a direct relationship to the abundance and

76 richness of natural enemies in the same landscapes (Letourneau et al. 2009).

77 Effects of natural enemies on pests can involve multiple interactions that prevent them

78 from being deducible from the diversity of the natural enemy community (Rand et al. 2012;

79 Martin et al. 2013). These interactions can be additive or synergistic, i.e. pest control by multiple

80 enemies is as strong or stronger than the sum of each enemy's effect in isolation; neutral, i.e. pest

81 control is similar whether enemies are combined or not; or antagonistic, i.e. negative interactions

82 such as intraguild predation or behavioral interference lead to lower pest control when enemies

83 are combined than in isolation (Letourneau et al. 2009). These effects are determined by a

84 variety of possible mechanisms, including niche complementarity and functional redundancy in

85 addition to intraguild predation (Straub et al. 2008; Tylianakis and Romo 2010), and may also be

86 affected by the complexity of local vegetation structures (Janssen et al. 2007). However, in

87 terrestrial systems, interactions of predator species and functional groups have almost

88 exclusively been investigated at small spatial and temporal scales that do not reflect the

89 complexity of real-world landscapes (Duffy et al. 2007; but see Thies et al. 2011; Holland et al.

90 2012). Results of these local studies show highly unpredictable interactions across systems and

91 organisms, and little consensus has been reached on the factors that determine interaction 
92 strength and direction in real-world ecosystems (Tylianakis and Romo 2010). Thus, although

93 pest control requires the presence of natural enemies in the system, its effectiveness can only be

94 approximated by simple measures of enemy community composition (abundance, species

95 richness) if the direction of enemy interactions is known across all relevant spatial and temporal

96 scales. Quantifying effective pest control across landscapes thus requires measuring the effects

97 of the entire pool of enemies; predicting variations of these effects requires identifying the

98 individual contributions of different enemies to pest control, and the nature of their interactions

99 across landscapes (Martin et al. 2013). For this, definition of complexes of natural enemies

100 (guilds) of similar body size, mobility and foraging habits is a meaningful and, importantly,

101 realistically applicable proxy (Macfadyen et al. 2014).

102 Aphids are a major pest of crops worldwide. Several groups of natural enemies are

103 known to provide control of aphid pests (Schmidt et al. 2003). They include parasitoid wasps and

104 syrphid fly larvae ("flying insects"), which colonize aphid-infested plants by flying onto them as

105 adults and ovipositing in or near aphids. More generalist enemies include carabid and staphylinid

106 beetles and spiders, which mainly colonize plants from the ground and occupy a lower stratum

107 than flying insects ("ground-dwellers"; Schmidt et al. 2003). In addition, birds represent the top

108 predators for insects in many agricultural systems. Direct effects of bird predation on aphids

109 have rarely been assessed (but see Tremblay et al. 2001), but their potential role as antagonists of

110 other enemies may seriously impact overall amounts of pest control (Martin et al. 2013).

111 In this study, we use multiple natural enemy exclosures on pests of cabbage Brassica

112 oleracea var. capitata in a replicated design across landscapes of an agricultural region in South

113 Korea. We examined the effects of three main functional guilds of natural enemies on pest

114 control of aphids across a gradient in landscape complexity. The three enemy functional guilds 
115 distinguished here are birds and other vertebrates larger than $1.5 \mathrm{~cm}$; flying insects including

116 syrphid flies, parasitoid and predatory wasps; and ground-dwellers including spiders, carabid and

117 staphylinid beetles. Specifically, we aimed 1) to assess the strength of pest control by all natural

118 enemies combined on aphid populations, across a gradient in landscape complexity; 2) to

119 identify the separate contributions of functional guilds of natural enemies to pest control at the

120 landscape scale and the nature of enemy interactions across landscapes and 3) to evaluate the

121 importance of local management intensity for these effects. We hypothesized that interactions of

122 the natural enemy pool may change with landscape complexity due to changing amounts of

123 seminatural habitat as a refuge against intraguild predation (Janssen et al. 2007), contiguity of

124 more distinct habitats intensifying spillover, and changing density ratios between pests, enemies,

125 and among enemy functional guilds (Bianchi et al. 2006; Chaplin-Kramer et al. 2011); and that

126 this has consequences for overall pest control strength (Martin et al. 2013).

128 Materials and Methods

129 1. Study sites and landscapes. This experiment was performed from July to September 2010 in

130 the $55 \mathrm{~km}^{2}$ agricultural region of Haean, South Korea (long. $128^{\circ} 5^{\prime}$ to $128^{\circ} 11^{\prime} \mathrm{E}$, lat. $38^{\circ} 13^{\prime}$ to

$13138^{\circ} 20^{\prime} \mathrm{N}$; Supplementary material Fig. S1). This region is located at the head of the Soyang Lake

132 watershed, a major water and energy source for the northern half of South Korea. In this region,

133 annual and perennial crops are cultivated in fields $<1$ ha separated by seminatural margins.

134 Patches of deciduous forest and riparian corridors are distributed throughout, contributing to high

135 landscape heterogeneity.

136 Eighteen fields were selected in this region (Martin et al. 2013), including 16 fields inside

137 the Haean catchment (Fig. S1) and two fields $20 \mathrm{~km}$ to the south, in an area with similar land use 
138 and climatic conditions. Of these, 13 were managed organically and five conventionally. Due to

139 the difficulty of convincing farmers to perform experiments in their fields, the number of organic

140 vs. conventional fields could not be balanced in this design. Nine fields were planted with a

141 Brassicaceae crop and nine with one of five other crop families. Planting dates of these crops

142 varied from 0 to 55 days $(22 \pm 20$, mean $\pm \mathrm{SD})$ before the start of the experiment. Fields were

143 distant by a minimum of $211 \mathrm{~m}$ (distance from field edge), with a mean inter-field distance of

$1443.2 \pm 0.1 \mathrm{~km}($ mean $\pm \mathrm{SE})$. The field locations covered a gradient in landscape complexity from

$1456.3 \%$ to $43.3 \%$ seminatural habitat in a $700 \mathrm{~m}$ radius around fields (see below), and bordered on

146 seminatural margins of similar size and plant composition. Seminatural habitat was defined in

147 this region as including seminatural field margins, secondary regrowth and shrubs, 1 and 2-year

148 old fallows, and forest edges ( $2 \mathrm{~m}$-wide buffers). Its proportion around fields was calculated

149 using polygon maps of the region (ground-truthed in 2009 and 2010; Seo et al. 2014) in ArcGIS

$150 \quad 9.3$ and R Statistical Software v. 2.13.1 (R Core Team 2013).

151 2. Experiment. Within each field, one $20 \mathrm{~m}^{2}$ plot was reserved for the experiment and left

152 uncropped. It was separated from the surrounding crop by a plastic barrier and planted with

153 cabbage Brassica oleracea var. capitata. No pesticides were applied on these plots. After an

154 initial 20 days, six rows of four cabbages were randomly marked in each plot from which all

155 herbivores were removed. Six natural enemy exclusion treatments were installed on these plants

156 between day 20 and 21 and maintained until harvest after 60 days.

157 We initialized the experiment by inoculating aphids in these treatments. In order to enable

158 comparison between the six treatments of a given plot, the same starting number of aphids was

159 inoculated in all the treatments of one plot (Chaplin-Kramer and Kremen 2012). This was done

160 by placing on each treated cabbage the average number of aphids per plant found in the plot. As 
161 densities were low at the start of the season, aphids thus inoculated in each plot varied from 2 to

16210 per plant. In plots where no aphids were present at the beginning of the experiment, a

163 minimum of two adult aphids per plant was inoculated. This procedure was selected in

164 preference to inoculation of the same number of aphids in all plots, in order to increase

165 representativeness in the experiment of the pest pressure occurring in each plot (Thies et al.

166 2011). Cabbage leaves infested with laboratory-reared cabbage aphids Brevicoryne brassicae

167 (Linné) were transported to the field and fragments with the approximate number of aphids were

168 deposited on treated cabbages. After one day, successful transfer of the desired number of aphids

169 to treated plants was verified and any aphids in excess were removed. Initial aphid densities were

170 unrelated to either the percent seminatural habitat around fields (Poisson linear model corrected

171 for overdispersion, $\mathrm{n}=18, \mathrm{t}=0.3, \mathrm{p}=0.8)$, the crop type of surrounding fields $(\mathrm{t}=1.2, \mathrm{p}=0.2)$ or their

172 management $(t=-0.3, p=0.8)$. Initial densities tended to be positively related to the maturity of the

173 surrounding crop, but the relationship was not significant $(\mathrm{t}=1.9, \mathrm{p}=0.08)$.

174 Starting 10 days after initializing the treatments, arthropods were monitored at three

175 occasions (10 day intervals) throughout the growth period, by carefully inspecting both sides of

176 leaves and recording the number, species and life stage of arthropod herbivores, parasitoids

177 (parasitized aphid mummies) and predators, mainly the larvae of syrphid flies. Sap-sucking

178 species included cabbage aphids Brevicoryne brassicae, green peach aphids Myzus persicae

179 (Sulzer) and low densities of the turnip aphid Lipaphis erysimi (Kaltenbach). Larvae of the leaf-

180 chewing Lepidoptera Pieris rapae, Pieris brassicae (Linné) and Trichoplusia ni (Hübner) were

181 also present and their effects are described in a separate publication (Martin et al. 2013); see

182 below). After 60 days, cabbage plants were harvested and weighed for fresh biomass. As one

183 plot was monitored on two occasions only and monitoring data from one plant are missing, the 
184 total number of observations is 17 plots $\times 6$ treatments $\times 4$ plants $\times 3$ sampling dates +1 plot $\times 6$ 185 treatments $\times 4$ plants $\times 2$ sampling dates -1 plant $=1271$. Whereas Martin et al. (2013) present

186 results for Lepidopteran pests only, the present study focuses on the response of aphid pests. The

187 final biomass of cabbages is the only measure common to both studies, and is examined here in 188 relation to aphids and their enemies.

189 3. Field exclusion treatments. Natural enemy exclusion treatments were cages designed to 190 exclude combinations of three guilds of natural enemies: G - ground-dwellers (spiders, carabid 191 and staphylinid beetles), F - flying insects (syrphid flies, parasitoid and predatory wasps), and B -

192 birds and other vertebrates larger than $1.5 \mathrm{~cm}$. Cages were $50 * 150 * 100 \mathrm{~cm}$ and covered one row 193 of four cabbage plants. We used combinations of chicken wire (1.5 $\mathrm{cm}$ mesh size $)$, fine polyester 194 mesh $(0.8 \mathrm{~mm})$ and plastic barriers coated with insect glue to exclude either all enemies 195 (treatment -G-F-B, "no enemy" control), birds and flying insects (-F-B), ground-dwellers and 196 birds (-G-B), only ground-dwellers (-G), only birds (-B), or no enemies (O, open control). Cage 197 treatments and corresponding symbols are summarized in Figure S2. Although designed to 198 exclude or allow access to specific enemy guilds with relevance for pest control, these treatments 199 do not allow to distinguish the effect of increasing enemy diversity per se, vs. increasing enemy 200 density due to access by a higher number of guilds (additive design sensu Tylianakis and Romo 201 2010). However, they do provide insight on the relative importance of separate guilds, and on the 202 interactions taking place between guilds in terms of their outcome for pest control (Schmidt et al. 203 2003).

204 An additional treatment excluding both enemies and herbivores controlled for abiotic soil 205 conditions between plots; ecofriendly pesticide was applied at the start of the experiment in this 206 treatment only. Differences in soil conditions between plots had no effect on final cabbage 
207 biomass (Martin et al. 2013). Although fine mesh cages were effective at excluding most natural 208 enemies including parasitoids, they were not impermeable to external colonization by aphids, as 209 indicated by test cages without inoculation (E. Martin, pers. obs). At the start of the experiment, 210 two live pitfall traps were installed in all treatments excluding ground-dwellers. After initial 211 collection of the ground arthropods already present, pitfall traps remained empty throughout the 212 experiment. This method was effective also for spider exclusion (Martin et al. 2013).

213 Microclimatic and light differences between treatments were tested by comparing values inside 214 and outside fine mesh treatments in each plot (Martin et al. 2013). Light transparency of fine 215 mesh treatments was $83 \pm 0.9 \%$ (mean \pm s.e.m). Air humidity did not differ significantly between 216 the inside and outside of cages $(t=1.4, p=0.3$ and $t=0.04, p=0.7$ on sunny and rainy days, 217 respectively). Temperatures were $0.56 \pm 0.1^{\circ} \mathrm{C}$ higher inside than outside fine mesh treatments 218 (mean \pm s.e.m; $\mathrm{t}=5.5, \mathrm{df}=17, \mathrm{p}<0.001)$. However, microclimatic and light differences had no 219 significant effect on plant growth between treatments (Martin et al. 2013). In addition, any error 220 caused by these differences would have occurred in all plots, and thus should not affect result 221 interpretation at the landscape scale. As only $2.2 \%$ of aphids were winged ( 700 out of 31,503 222 counted individuals), the role of aphid dispersal appears to have been negligible (Thies et al. 223 2011).

224 4. Data analysis. Aphid population growth, parasitism rate, syrphid fraction and final crop 225 biomass were analyzed using linear and generalized linear mixed models in R Statistical 226 Software 2.13.1 (R Core Team 2013).

227 Average daily aphid population growth ( $\mathrm{n}=1271$ data points; Methods §2) was calculated as the $228 \log (\mathrm{N}+1)$-ratio per day of aphid densities (sampling dates 1 to 3 ) to initial densities. For example, 229 at sampling date 1 , aphid population growth was $\left[\log \left(\mathrm{N}_{\text {aphids at date } 1}+1\right)-\log \left(\mathrm{N}_{\text {aphids }}\right.\right.$ 
initial $\left.\left.^{+1}\right)\right] /($ number of days from the start of the experiment to date $1=10)$. At date 2 , it was

$231\left[\log \left(\mathrm{N}_{\text {aphids at date } 2}+1\right)-\log \left(\mathrm{N}_{\text {aphids initial }}+1\right)\right] /($ number of days from the start of the experiment to

232 date $2=20$ ). These measures of population growth thus reflect either relatively short-term (until

233 date 1, 10 days), mid-term (until date 2, 20 days) or long-term (until date 3, 30 days) changes in 234 aphid populations over time.

235 Here, as in other studies quantifying aphid pest control on a landscape gradient (e.g.

236 Holland et al. 2012; Rusch et al. 2013; Thies et al. 2011; but see Chaplin-Kramer and Kremen

237 2012), the calculation of aphid density and population growth accounts in practice for both

238 processes of growth and colonization from the surrounding area. In this study, colonization

239 occurred in all treatments (see Methods §3). Although differences in colonization between

240 treatments could not be quantified, any hindrance of colonization by fine mesh cages would lead

241 to an underestimation, not an overestimation, of actual pest control. Indeed, if treatments

242 accessible to enemies were more colonized than fine mesh controls, then enemies appear to have

243 reduced aphids less than they "truly" have (final densities after predation = remaining aphids

244 after predation + additional colonized aphids). Here, aphid population growth (defined as the

245 outcome of growth and colonization by all aphid species) was used as a response variable instead

246 of aphid density, in order to account for a variable number of aphids initially present in each plot

247 (Rusch et al. 2013; Thies et al. 2011).

248 Aphid population growth was modelled using a linear mixed model in package nlme

249 (Pinheiro et al. 2013) and variance functions were included to model heteroscedasticity.

250 Parasitism rates (the ratio of parasitized to all aphids) and syrphid fractions (the ratio of syrphids

251 to total aphids + syrphids) were modelled using a binomial response with logit link in package

252 lme4 (Bates et al. 2014). Observation-level random effects were included to account for 
253 overdispersion (final overdispersion parameter $\Psi<0.2$ ). Crop biomass ( $\mathrm{n}=432$ plants) was

254 modelled using a Gamma error with log link in package lme4. All models included 'exclusion

255 treatment' (6 levels of natural enemy exclusion) nested within 'plot' (18 plots, each in one

256 landscape sector) as random effects, in order to account for pseudoreplication within each plot

257 and exclusion treatment. Thus, all plants within the same exclusion treatment are treated as non-

258 independent replicates of that treatment. For all responses except biomass (see below),

259 explanatory variables included exclusion treatment, percent seminatural habitat in a radius

260 around each plot, sampling date (1-3), management type of the surrounding field (organic /

261 conventional), crop type (Brassicaceae / non Brassicaceae) and crop maturity of the surrounding

262 field, and 2-way and 3-way interactions. Management, crop type and crop maturity did not

263 correlate significantly either with percent seminatural habitat or with each other (Pearson's $r$

264 always $<0.3, \mathrm{p}>0.2$ ).

265 Aphid population growth and pest control may have been influenced not only by

266 environmental variables, but also by local aphid densities occurring in each plot. In order to

267 identify density-dependent effects of the number of aphids present, a second set of models was

268 constructed with the additional explanatory variable 'initial number of aphids' and interactions

269 with 'sampling date' and 'treatment'. The initial number of aphids for a given sampling date was

270 the number of aphids counted at the previous date (dates 2 and 3), or the number of aphids

271 initially inoculated (date 1 and for average daily aphid population growth). The initial number of

272 aphids was selected in $95 \%$ model confidence sets (see below) with a probability of 1 for aphid

273 population growth, 0.93 for parasitism and 0.29 for syrphid fractions (Table S1). Effects of the

274 initial number of aphids on aphid growth were negative for all sampling dates (averaged $b=-$

$2750.006 \pm 0.002)$. Effects on parasitism were positive in treatments accessible to parasitoids 
$276(b=0.46 \pm 0.42$ in $-G-B)$, and slightly positive on syrphid fractions $(b=0.003 \pm 0.055)$. Results of

277 other explanatory variables and their interpretation were not affected by inclusion of this factor,

278 thus the initial number of aphids was not included in subsequent analyses (Table S1).

279 In order to determine the most adequate spatial scales for analysis of each response

280 variable, Akaike's Information Criterion with a correction for finite sample sizes (AICc) was

281 used to compare the final models at $100 \mathrm{~m}$-intervals between $100 \mathrm{~m}$ and $1000 \mathrm{~m}$ around fields.

282 Lowest AICc values were selected at $700 \mathrm{~m}$ for aphid population growth, $200 \mathrm{~m}$ for parasitism

283 rates and $900 \mathrm{~m}$ for syrphid fractions. Results are shown for these most predictive scales.

284 However, effects of landscape and landscape:treatment interactions were also selected with

285 similar effects in model confidence sets (see below), at all but three other scales for aphid

286 population growth (Table S2).

Model selection was performed by assembling a $95 \%$ confidence set of models

288 (cumulated sum of AICc weights $\leq 95 \%$ ) from the set of all possible models, using sequential

289 AICc testing with the function "dredge" in R package MuMIn (Barton 2012). Model averaging

290 was performed on this set and weights were calculated for each explanatory variable as the sum

291 of the AICc weights of each model it occurs in. Weights of each explanatory variable can thus be

292 interpreted as the probability of its presence, or importance, within the global averaged model

293 (Burnham et al. 2011). Model-averaged coefficients of explanatory variables were used to plot

294 the predicted values of responses. Tukey multiple comparisons of means were performed on

295 models without interactions, and slope comparisons of models with interactions were performed

296 using manually defined contrast matrices. P-values of multiple comparisons were adjusted for

297 the False Discovery Rate (Benjamini and Yekutieli 2001). Models were checked graphically for

298 violation of assumptions of normality and homoscedasticity. Spline correlograms of Moran's I 
299 against distance confirmed that any spatial autocorrelation present in the raw data (for instance

300 due to overlapping landscape sectors) was accounted for by inclusion of the model random

301 effects (Zuur et al. 2009), thus potential non-independence of sites due to spatial proximity was

302 accounted for in models. Further, temporal autocorrelation due to non-independence of sampling

303 dates was addressed by including a correlation structure in models for aphid population growth

304 of the form corAR1( Date $\mid$ Plot_ID/Treatment_ID/Plant). In generalized mixed models in

305 lme4, such structures are not yet implemented. For parasitism rates and syrphid fractions, we

306 thus accounted for effects of repeated measures by including 'sampling date' as an additional

307 random effect nested in 'exclusion treatment' and 'plot'. Results for aphid population growth and

308 syrphid fractions were unchanged by inclusion of these structures. For parasitism rates, they led

309 to a difference in the scale of response (200 $\mathrm{m}$ instead of $1000 \mathrm{~m}$ ) but to no change in result

310 interpretation. Thus, effects were considered robust for all response variables to possible effects

311 due to non-independence of sampling dates.

312 Ultimately, the final measure of pest control for farmers is the biomass of the crop. In this

313 experiment, final crop biomass decreased with increasing landscape complexity in all treatments

314 except -G-B, reflecting the impact of stronger herbivory by Lepidopteran pests in complex

315 landscapes, as previously shown elsewhere (Pearson's $\mathrm{r}=-0.53, \mathrm{P}<0.001$ between biomass and

316 herbivory by Lepidoptera; Martin et al. 2013). In order to estimate, in turn, the importance of

317 aphid suppression for final biomass provision, we used a separate set of models relating aphid

318 population growth and final crop biomass, respectively, to syrphid fractions and parasitism rates.

319 Tested effects of aphid population growth on biomass were not modified by inclusion of

320 Lepidopteran herbivory in models. 

compared to controls without enemies were calculated from model predicted values as $\left(\mathrm{R}_{\text {treatment }}{ }^{-}\right.$

$323 \mathrm{R}_{\text {control }} / \mathrm{R}_{\text {control }}$ where $\mathrm{R}=$ mean aphid population growth in all landscapes.

\section{Results}

Effects of enemy guilds and landscape context on aphid pest control

327 On the first sampling date, aphid densities averaged $44.3 \pm 6.2$ individuals / plant (minimum 0, maximum $1105 ; \mathrm{n}=1271$ plants in 18 plots). Mean densities decreased in the following dates to $20.6 \pm 4.4$ (min. 0, max. 1093) and $8.6 \pm 2.3$ (min. 0, max. 503) aphids / plant on dates 2 and 3, respectively. These were reflected by average population growth rates, which were positive in some treatments after 10 days (sampling date 1) but negative or zero over the whole season (30 332 days, sampling date 3; Fig 1).

333 On average, daily aphid population growth was four times (range 1-6 times) higher in the

334 absence of all natural enemies than in their presence (Fig. 1; Table 1). This effect was maintained across sampling dates, with overall population growth lowest in treatments accessible to natural

336 enemies until the end of the season. Individual enemy guilds reduced aphid population growth to

337 lower values than in their absence: on average, growth rates were 2.3 times (0.2-4) and 3.3 times 338 (0.9-5) higher in the absence of ground-dwellers and flying insect enemies respectively, than in 339 their presence individually. Direct effects of vertebrate predators (birds) are not measurable in 340 isolation from flying insect enemies. However, the exclusion of birds from treatments with other 341 enemy guilds did not significantly impact mean aphid population growth at any date (O vs. -B 342 and -G vs. -G-B; Fig. 1). 
344 stronger than in isolation (Fig. 1). This result was particularly present in the first phase of the 345 experiment (until date 1), and lessened over time (dates $2 \& 3$ ). On average, population growth 346 was 1.9 times (1.4-2.6) higher with ground-dwellers alone than in the presence of both guilds, 347 and 0.8 times $(0.1-1.7)$ higher with flying insects alone than in the presence of both guilds.

348 Overall, ground-dwellers and flying insects thus had complementary impacts on aphid 349 suppression. Aphid population growth in controls excluding all natural enemies increased from simple to complex landscapes (Figs 1, S3; Table S3: -G-F-B vs. zero, $p_{\text {adjusted }}<0.05$ for sampling dates 1 landscape complexity (landscape:treatment interaction; Tables 1, S3), and these effects were maintained across several spatial scales (Table S2). At the $700 \mathrm{~m}$ scale around fields, aphid suppression by all natural enemies (the difference between population growth in the absence and in the presence of all enemies) was ca. six times higher in complex than in simple landscapes (mean \pm s.e.m. across sampling dates $5.6 \pm 2.5$, from a landscape with $18 \%$ to a landscape with $45 \%$ seminatural habitat; Table S3: the slope of O is significantly lower than the slope of -G-F-B at dates 1 and 2, thus the difference between $\mathrm{O}$ and -G-F-B increases with landscape complexity). This effect was maintained in treatments combining multiple guilds (-G, -B).

361 Further, effects of ground-dwellers only (the difference between treatments with ground-dwellers

362 only and controls without enemies) were less strong across the landscape gradient than effects of

363 flying insects (Table S3: -G-F-B vs. -F-B and vs. -G-B). In complex landscapes, flying insect

364 enemies reduced aphid growth ca. two times more than ground-dwelling predators (mean \pm s.e.m. 
365 across sampling dates $2.1 \pm 0.4$; reduction compared to controls for flying insects and ground-

366 dwellers, respectively).

367

368 Parasitism rates and syrphid fractions

369 Rates of parasitism and syrphid fractions were higher in treatments accessible to flying insects

370 than in treatments excluding them, confirming the effectiveness of exclosures for these enemies

371 (Fig. 2, Table 1). These differences were significant for parasitism rates and less strong for

372 syrphid fractions, as only low numbers of syrphid larvae (on average $0.3 \pm 0.03$ in accessible

373 treatments) were recorded per plant. Rates of parasitism and syrphid fractions increased with

374 landscape complexity mainly in treatments accessible to flying insects only (Fig. 2; Table S3: -

375 G-B vs. zero). Although reduction of aphid population growth was strongest in complex

376 landscapes when flying insects were combined with other guilds (Fig. 1), this was not reflected

377 by similar high parasitism or syrphid fractions in complex landscapes in treatments combining

378 several guilds (Fig. 2). This difference thus suggests a negative effect of bird and ground-dweller

379 access on the effectiveness of flying insect enemies.

380

381

Aphid population growth and yields

382 Aphid population growth was strongly negatively correlated with parasitism rate and syrphid

383 fractions (Fig. 3), confirming the impact of these enemies for reduction of aphid populations.

384 Neither population growth nor cumulated aphid densities led to a significant decrease in final

385 crop biomass. However, high syrphid fractions tended to have a positive impact on biomass, thus

386 indicating a link via pest suppression between the proportion of syrphid predators and the

387 provision of yields (Fig. 3). This is reinforced by a significantly positive link between biomass 
388 and syrphid densities $\left(\mathrm{Chi}^{2}=4.5, \mathrm{p}=0.03, \mathrm{n}=431\right)$. In contrast, neither parasitism rate nor

389 parasitoid density significantly affected crop biomass $\left(\mathrm{Chi}^{2}=0.6, \mathrm{p}=0.4\right.$ and $\mathrm{Chi}^{2}=2, \mathrm{p}=0.2$,

390 respectively).

391

392 Management effects

393 After the start of the experiment, neither the crop type of surrounding fields (Brassicaceae vs.

394 non Brassicaceae) nor their maturity had an impact on aphid population growth or enemy rates

395 (Table 1). However, the management intensity of surrounding fields (organic vs. conventional)

396 affected response variables. Particularly at sampling date 1, population growth was higher in

397 plots surrounded by conventional than by organic fields (Fig. S3; Table 1). However, no

398 differences were found at subsequent dates (Fig. S3). Aphid populations thus decreased more

399 strongly from date 1 to date 3 in fields surrounded by conventional than by organic fields. In

400 contrast, enemy densities and parasitism rates were similar in both management types at date 1 ,

401 but higher near conventional fields on the following dates (Figs. S4, S5).

402

403 Discussion

404 Pest control across the landscape gradient

405 This study shows that in the absence of pressure by natural enemies, aphid population growth

406 was higher in complex than in simple landscapes. However, aphids were also strongly reduced

407 by natural enemies, and this pest reduction increased with landscape complexity. Overall, aphids

408 were thus maintained at low levels in all landscapes, because higher aphid growth and

409 colonization in complex landscapes was compensated by stronger pest control by natural

410 enemies. 
An increase of aphid population growth, but also of aphid suppression with landscape

412 complexity can be explained by higher availability of alternative resources and overwintering

413 habitats in seminatural areas around fields, which may benefit colonization and reproduction in

414 fields by both pests and their natural enemies in the course of the growing season (Thies et al.

415 2005; Bianchi et al. 2006; Holzschuh et al. 2010). Here, stronger aphid population growth was

416 found in complex landscapes in treatments excluding natural enemies, where aphids were

417 enclosed in fine mesh cages as a barrier to natural enemies. Such higher growth rates may have

418 been caused by an additional factor such as differences in the nitrogen content of fields (Butler et

419 al. 2012). In this study, known management differences did not correlate with landscape context,

420 but interactions of these factors could not be tested due to a lower range of landscape values

421 surrounding conventional fields. In addition, cages were permeable to some extent to

422 colonization by aphids (see Methods). Although densities in plots at the start of the experiment

423 were not predicted by the landscape context, aphid colonization is likely to have taken place in

424 the course of the experiment, particularly in the first phase of the growing season (until sampling

425 date 1). Thus, a positive impact of seminatural habitats on aphid colonization is the most

426 plausible explanation for higher aphid growth rates in complex landscapes in the absence of

427 enemies.

428 Several studies have measured the distribution of pest abundance across landscapes, but

429 results remain inconclusive overall, as pests appear to decrease, increase or not to vary with

430 landscape complexity (Bianchi et al. 2006; Chaplin-Kramer et al. 2011). However, only a few

431 recent studies have measured landscape effects on pests in the absence vs. in the presence of

432 natural enemies and thus provide measures of actual pest pressure across landscapes (Gardiner et

433 al. 2009; Thies et al. 2011; Chaplin-Kramer and Kremen 2012; Holland et al. 2012; Martin et al. 
434 2013; Rusch et al. 2013). Of these, three report results of pest variation separately from an

435 aggregated measure of pest control, with pest pressure either increasing with landscape

436 complexity as here (Chaplin-Kramer and Kremen 2012; Martin et al. 2013) or decreasing with

437 combined landscape and local extensification (Thies et al. 2011). The lack of general patterns

438 highlights the fact that pest densities are affected by the landscape both directly and indirectly

439 through landscape effects on higher trophic levels (enemies) and emphasizes the need to

440 experimentally address these factors in isolation from each other.

441 In agreement with our results, the few studies measuring actual pest control of aphids as the

442 difference between pest density in the presence and in the absence of natural enemies, also find

443 increasing intensity of pest control with the proportion of natural or seminatural habitats in the

444 surrounding landscape. The strength of aphid pest control increased in these studies by a factor

445 of two to five from simple to complex landscapes (Gardiner et al. 2009; Chaplin-Kramer and

446 Kremen 2012; Rusch et al. 2013), and was here on average six times higher in complex than in

447 simple landscapes. So far, however, no other study has disentangled the single and combined

448 contributions of antagonist guilds including birds to aphid pest control along a landscape

449 complexity gradient.

450

451 Enemy contributions to pest control and interactions

452 On average, our results suggest that flying insect enemies had stronger impacts than ground-

453 dwellers on aphid control. Further, these effects appeared to increase in complex landscapes,

454 paralleling the generally higher abundance and species richness of these enemies in landscapes

455 with high amounts of (well-connected) seminatural habitat, than in simple ones where

456 overwintering, nesting, and food resources are rare (Bianchi et al. 2006; Holzschuh et al. 2010; 
457 Chaplin-Kramer et al. 2011). The importance of landscape complexity for flying insect

458 effectiveness is confirmed by increased activity rates (parasitism and syrphid fractions) of this

459 guild in complex landscapes, both here and in other studies (e.g. Thies and Tscharntke 1999;

460 Rand et al. 2012). However, increased parasitism of aphids is likely to be accompanied by even

461 stronger hyperparasitism in complex landscapes, because sensitivity of these organisms to the

462 landscape context has been shown to increase with trophic level (Rand et al. 2012). The

463 parasitism rates observed here thus likely reflect the outcome of interactions with the $4^{\text {th }}$ trophic

464 level in the previous year, and indicate that parasitism was maintained in complex landscapes

465 despite possible pressure by hyperparasitoids.

466 Control by ground-dwellers increased to a lesser extent with landscape complexity than control

467 by flying insect enemies. As a result, the relative contribution of these guilds to pest suppression

468 was influenced by the landscape context. Stronger effects of flying insects compared to ground-

469 dwellers in complex landscapes are in agreement with previous studies (Schmidt et al. 2003;

470 Thies et al. 2011; but see Safardoza et al. 2014) and with the idea that generalist predators

471 (ground-dwellers) have lower impacts than specialists when prey density is high (Straub et al.

472 2008). Indeed, within our study system, relative prey densities were highest in these landscapes.

473 Birds, in contrast, showed no clear contribution to reducing aphids. Thus, in addition to generally

474 showing predictable responses to changes in landscape complexity (Bianchi et al. 2006), the

475 guild of flying insects had the strongest impact on aphids under conditions of high landscape 476 complexity.

477 Strong impacts of flying insects are confirmed by the negative relationships between 478 aphid population growth rates and parasitism and syrphid fractions. However, of these, only 479 syrphids had a positive impact on crop biomass, the final measure of interest for assessment of 
480 pest control (in this experiment, landscape effects on biomass were linked to the Lepidopteran

481 pest complex; Martin et al. 2013). Ultimately, benefits for farmers may be higher when enemies

482 are predators that immediately suppress pests, than when they are parasitoids with slower

483 impacts on their hosts. Overall, flying insect enemies and particularly syrphid flies may thus

484 represent an optimal focus for efforts to maximize natural pest control in agricultural landscapes.

485 However, the effectiveness of these efforts depends on the balance between individual enemy

486 contributions, and how they interact with other natural enemy guilds.

487 Interactions between flying insects and ground-dwellers led to complementary effects on

488 pest control, as aphid suppression was stronger in the presence of both guilds than with either

489 guild alone, in agreement with results in other aphid systems (Safardoza et al. 2014, Schmidt et

490 al. 2003). This effect may be due to density-dependent predation by each guild, to their spatially

491 segregated foraging (Straub et al. 2008), but also to escape behavior of aphids from flying insects

492 increasing the chances of ground-dweller predation (Losey and Denno 1998). In this system,

493 however, escape behavior of the aphids was neither observed in the field (pers. obs.) nor appears

494 to be documented for the species considered; in contrast, several species of ground-dwellers

495 present in this region (spiders, carabids and staphylinids) are known to forage by climbing

496 directly onto crop plants (Hannam et al. 2008; Suenaga and Hamamura 1998). In the absence of

497 antagonist interactions, access to both guilds may increase overall enemy density and thus

498 benefit aphid control. Syrphid fractions show that syrphid larvae were little influenced by the

499 presence of ground-dwellers and could thus suppress aphids independently of ground-dweller

500 activity. Lower parasitism in treatments accessible to ground-dwellers indicate that in complex

501 landscapes, ground-dwellers may have preyed not only on live aphids, but also or preferentially 
502 on parasitized mummies, as observed in local-scale studies (Snyder and Ives 2001). However,

503 this did not hinder the overall complementarity of these guilds for aphid suppression.

504 Birds, the largest and most generalist predators in the system, appeared to interact in

505 complex ways with aphids and other enemies. Although their effects have rarely been quantified

506 in agricultural systems with annual crops (Mooney et al. 2010) and almost never in the light of

507 interactions with other enemies (but see Hooks et al. 2003; Martin et al. 2013), birds are known

508 to occasionally feed on aphids in these systems (Tremblay et al. 2001). Here, predation by birds

509 of parasitized aphids and syrphids in complex landscapes is suggested by lower enemy rates in

510 bird-accessible treatments, at least while aphid populations are high (sampling dates $1 \& 2$ ). Bird

511 predation on parasitoids vs. syrphids has different consequences: coincidental predation of

512 mummies may still decrease aphid densities, but omnivorous predation of syrphids should

513 theoretically release the shared prey (Straub et al. 2008). Overall, effects on aphids may thus

514 level out and, as found here, lead to no clear effect of birds on aphid population growth.

515 Overall, strong negative effects of intraguild predation on aphid suppression were not

516 found, as aphids were reduced sufficiently by the combination of all guilds to remain at low

517 levels in open treatments throughout the experiment. This result is in contrast to the disruptive

518 effects of intraguild predation by birds found for Lepidopteran pest control in the same system

519 (Martin et al. 2013). It thus emphasizes that effects of natural enemies and particularly birds are

520 pest organism-dependent. In the case of aphids, pest control provided by the combination of

521 three enemy guilds was higher than pest control by individual guilds, and this result held true

522 across landscapes with increasing complexity. These results support the idea that higher

523 functional diversity may benefit ecosystem function and services across large spatial scales

524 (Cardinale et al. 2006), for particular combinations of functions and guilds. However, 
525 consideration of additional guilds or different pests may greatly influence this relationship, as

526 suggested by contrasting effects of enemy functional diversity on control of Lepidopteran pests

527 in the same experiment (Martin et al. 2013).

528 Interactions among pests, particularly between Lepidoptera and aphids, may also take

529 place that influence pest population growth, predation, parasitism and enemy-level interactions.

530 Though not testable by the present design, among-pest interactions merit further investigation,

531 particularly their potential response to variations in enemy density and community composition.

532

533 Natural enemies and pests in organic vs. conventional plots

534 In plots surrounded by conventional fields, soil nitrogen availability was higher than near organic

535 fields (Martin et al. 2013) and is likely to be responsible for higher initial aphid population build-

536 up in these in plots (Butler et al. 2012). More natural enemies than initially present were thus

537 required near conventional fields to effectively constrain aphids to the same degree as near

538 organic fields. Higher population growth at sampling date 1 in conventional fields, followed by

539 no differences at subsequent dates, suggest that a time lag took place near conventional fields

540 before enemies reached sufficient densities to effectively reduce pests in these plots (Krauss et

541 al. 2011). This supports the idea that early-stage pest control was less efficient near conventional

542 compared to organic fields. In contrast, strong pest regulation near conventional fields later in the

543 season indicates that enemies responded with strong positive density-dependence to the initial

544 population build-up of aphids in these fields, which may have been caused by the emission of

545 enemy-recruiting volatiles by the plants under herbivore attack (Kessler and Baldwin, 2001;

546 Thaler 1999). 


\section{Conclusion}

549 Despite complex interactions occurring between enemy functional guilds across landscapes, pest

550 control of aphids benefited in all landscapes from high enemy functional diversity, and was

551 stronger in complex landscapes with high amounts of seminatural habitat than in simple ones.

552 Aphid pest control by flying insects and ground-dwellers was complementary, but flying insects

553 including syrphids provided the strongest contributions to aphid pest control particularly in

554 complex landscapes. To our knowledge, this study is the first to provide results of natural enemy

555 interactions for aphid pest control outside of Europe and the USA. These results emphasize the

556 need to identify underlying interaction mechanisms of pest control at large spatial scales, in order

557 to provide realistic predictions of ecosystem service provision in agricultural landscapes

558 worldwide, and thus improve the applicability of this concept for higher agricultural

559 sustainability.

560

561 Acknowledgements

562 We thank the farmers of Haean and M. Ahn for permission to use their fields. J. Bae provided

563 translation and logistic help. M. Hoffmeister, G.-H. Im, P. Poppenborg and S. Lindner provided

564 field assistance.

565

566

References

567 Barton K. 2012. MuMIn: Multi-model inference. R package version 1.9.5.

568 Bates D, Maechler M, Bolker B. 2013. lme4: Linear mixed-effects models using S4 classes. R 569 package version 0.999999-2. 
570 Bengtsson, Ahnström, Weibull. 2005. The effects of organic agriculture on biodiversity and

571 abundance: a meta-analysis. Journal of applied ecology 42:261-269.

572 Benjamini Y, Yekutieli D. 2001. The control of the false discovery rate in multiple testing under 573 dependency. Annals of statistics 29:1165-1188.

574 Bianchi F, Booij C, Tscharntke T. 2006. Sustainable pest regulation in agricultural landscapes: a 575 review on landscape composition, biodiversity and natural pest control. Proceedings of the Royal 576 Society B: Biological Sciences 273:1715-1727.

577 Bommarco R, Kleijn D, Potts SG. 2013. Ecological intensification: harnessing ecosystem

578 services for food security. Trends in Ecology \& Evolution 28:230-238.

579 Burnham KP, Anderson DR, Huyvaert KP. 2011. AIC model selection and multimodel inference 580 in behavioral ecology: some background, observations, and comparisons. Behavioral Ecology 581 and Sociobiology 65:23-35.

582 Butler J, Garratt M p. d., Leather S r. 2012. Fertilisers and insect herbivores: a meta-analysis.

583 Annals of Applied Biology 161:223-233.

584 Cardinale BJ, Srivastava DS, Duffy JE, Wright JP, Downing AL, Sankaran M, Jouseau C. 2006.

585 Effects of biodiversity on the functioning of trophic groups and ecosystems. Nature 443:989586992.

587 Chaplin-Kramer R, O’Rourke ME, Blitzer EJ, Kremen C. 2011. A meta-analysis of crop pest and 588 natural enemy response to landscape complexity. Ecology Letters 14:922-932.

589 Chaplin-Kramer R, Kremen C. 2012. Pest control experiments show benefits of complexity at 590 landscape and local scales. Ecological Applications 22:1936-1948. 
591 Duffy JE, Cardinale BJ, France KE, McIntyre PB, Thébault E, Loreau M. 2007. The functional

592 role of biodiversity in ecosystems: incorporating trophic complexity. Ecology Letters 10:522593538.

594 Gardiner M, Landis D, Gratton C, DiFonzo C, O’Neal M, Chacon J, Wayo M, Schmidt N, 595 Mueller E, Heimpel G. 2009. Landscape diversity enhances biological control of an introduced 596 crop pest in the north-central USA. Ecological Applications 19:143-154.

597 Hannam JJ, Liebherr JK, Hajek AE. 2008. Climbing behaviour and aphid predation by Agonum 598 muelleri (Coleoptera: Carabidae). The Canadian Entomologist 140:203-207.

599 Holland JM, Oaten H, Moreby S, Birkett T, Simper J, Southway S, Smith BM. 2012. Agri-

600 environment scheme enhancing ecosystem services: A demonstration of improved biological

601 control in cereal crops. Agriculture, Ecosystems \& Environment 155:147-152.

602 Holzschuh A, Steffan-Dewenter I, Tscharntke T. 2010. How do landscape composition and 603 configuration, organic farming and fallow strips affect the diversity of bees, wasps and their 604 parasitoids? Journal of Animal Ecology 79:491-500.

605 Hooks CRR, Pandey RR, Johnson MW. 2003. Impact of avian and arthropod predation on 606 lepidopteran caterpillar densities and plant productivity in an ephemeral agroecosystem.

607 Ecological Entomology 28:522-532.

608 Janssen A, Sabelis MW, Magalhães S, Montserrat M, Van Der Hammen T. 2007. Habitat 609 structure affects intraguild predation. Ecology 88:2713-2719.

610 Kessler A, Baldwin IT. 2001. Defensive function of herbivore-induced plant volatile emissions

611 in nature. Science 291:2141-2144.

612 Krauss J, Gallenberger I, Steffan-Dewenter I. 2011. Decreased functional diversity and

613 biological pest control in conventional compared to organic crop fields. PloS one 6:e19502. 
614 Letourneau DK, Jedlicka JA, Bothwell SG, Moreno CR. 2009. Effects of natural enemy

615 biodiversity on the suppression of arthropod herbivores in terrestrial ecosystems. Annual Review 616 of Ecology, Evolution, and Systematics 40:573-592.

617 Losey JE, Denno RF. 1998. Positive predator-predator interactions: enhanced predation rates and 618 synergistic suppression of aphid populations. Ecology 79:2143-2152.

619 Losey JE, Vaughan M. 2006. The economic value of ecological services provided by insects.

620 Bioscience 56:311-323.

621 Macfadyen S, Gibson R, Polaszek A, Morris RJ, Craze PG, Planque R, Symondson WO.,

622 Memmott J. 2009. Do differences in food web structure between organic and conventional farms 623 affect the ecosystem service of pest control? Ecology Letters 12:229-238.

624 Martin EA, Reineking B, Seo B, Steffan-Dewenter I. 2013. Natural enemy interactions constrain 625 pest control in complex agricultural landscapes. Proceedings of the National Academy of 626 Sciences 110:5534-5539.

627 Meehan TD, Werling BP, Landis DA, Gratton C. 2011. Agricultural landscape simplification 628 and insecticide use in the Midwestern United States. Proceedings of the National Academy of 629 Sciences 108:11500-11505.

630 Mooney KA, Gruner DS, Barber NA, Van Bael SA, Philpott SM, Greenberg R. 2010.

631 Interactions among predators and the cascading effects of vertebrate insectivores on arthropod 632 communities and plants. Proceedings of the National Academy of Sciences 107:7335-7340.

633 O'Rourke ME, Rienzo-Stack K, Power AG. 2011. A multi-scale, landscape approach to 634 predicting insect populations in agroecosystems. Ecological Applications 21:1782-1791.

635 Pinheiro J, Bates D, DebRoy S, Sarkar D, the R Development Core Team. 2013. nlme: Linear 636 and Nonlinear Mixed Effects Models. R package version 3.1-110. 
637 Rand TA, van Veen FJF, Tscharntke T. 2012. Landscape complexity differentially benefits

638 generalized fourth, over specialized third, trophic level natural enemies. Ecography 35:97-104.

639 R Core Team. 2013. R: A language and environment for statistical computing [Internet]. Vienna, 640 Austria: R Foundation for Statistical Computing; 2013.

641 Rusch A, Bommarco R, Jonsson M, Smith HG, Ekbom B. 2013. Flow and stability of natural

642 pest control services depend on complexity and crop rotation at the landscape scale. Journal of 643 Applied Ecology 50:345-354.

644 Safarzoda S, Bahlai CA, Fox AF, Landis DA. 2014. The Role of Natural Enemy Foraging Guilds 645 in Controlling Cereal Aphids in Michigan Wheat. PloS one 9:e114230.

646 Schmidt MH, Lauer A, Purtauf T, Thies C, Schaefer M, Tscharntke T. 2003. Relative importance 647 of predators and parasitoids for cereal aphid control. Proceedings of the Royal Society of London. 648 Series B: Biological Sciences 270:1905-1909.

649 Seo B, Bogner C, Poppenborg P, Martin E, Hoffmeister M, Jun M, Koellner T, Reineking B, 650 Shope CL, Tenhunen J. 2014. Deriving a per-field land use and land cover map in an agricultural 651 mosaic catchment. Earth System Science Data 6:339-352.

652 Snyder WE, Ives AR. 2001. Generalist predators disrupt biological control by a specialist 653 parasitoid. Ecology 82:705-716.

654 Straub CS, Finke DL, Snyder WE. 2008. Are the conservation of natural enemy biodiversity and 655 biological control compatible goals? Biological Control 45:225-237.

656 Suenaga H, Hamamura T. 1998. Laboratory evaluation of carabid beetles (Coleoptera:

657 Carabidae) as predators of diamondback moth (Lepidoptera: Plutellidae) larvae. Environmental 658 entomology 27:767-772. 
659 Thaler JS. 1999. Jasmonate-inducible plant defences cause increased parasitism of herbivores. 660 Nature 399:686-688.

661 Thies C, Haenke S, Scherber C, Bengtsson J, Bommarco R, Clement LW, Ceryngier P, Dennis

662 C, Emmerson M, Gagic V, Hawro V, Liira J, Weisser WW, Winqvist C, Tscharntke T. 2011.

663 The relationship between agricultural intensification and biological control: experimental tests 664 across Europe. Ecological Applications 21:2187-2196.

665 Thies C, Roschewitz I, Tscharntke T. 2005. The landscape context of cereal aphid-parasitoid 666 interactions. Proceedings of the Royal Society B: Biological Sciences 272:203-210.

667 Thies C, Tscharntke T. 1999. Landscape structure and biological control in agroecosystems. 668 Science 285:893-895.

669 Tremblay A, Mineau P, Stewart RK. 2001. Effects of bird predation on some pest insect 670 populations in corn. Agriculture, ecosystems \& environment 83:143-152.

671 Tscharntke T, Rand TA, Bianchi FJ. 2005. The landscape context of trophic interactions: insect 672 spillover across the crop—noncrop interface. In: Annales Zoologici Fennici. JSTOR, 421-432.

673 Tuck SL, Winqvist C, Mota F, Ahnström J, Turnbull LA, Bengtsson J. 2014. Land-use intensity 674 and the effects of organic farming on biodiversity: a hierarchical meta-analysis. Journal of 675 Applied Ecology 51:746-755.

676 Tylianakis JM, Romo CM. 2010. Natural enemy diversity and biological control: Making sense 677 of the context-dependency. Basic and Applied Ecology 11:657-668.

678 Zuur AF, Ieno EN, Walker N, Saveliev AA, Smith GM. 2009. Mixed Effects Models and 679 Extensions in Ecology with R. Springer US. 680 681 
684 Table

685 Table 1. Results of model selection relating landscape complexity and enemy exclusion to 686 response variables. Model lists show the $95 \%$ best models at the most predictive scale for each 687 response variable. w: AIC weight compared to all possible models, w95\%: AIC weight within 688 the $95 \%$ model confidence set. The sum of weights for each term is the sum of AIC weights of 689 all models selecting it and represents the probability of being present in the $95 \%$ model 690 confidence set. Selected explanatory variables are M: management of the nearest surrounding 691 field (organic / conventional); L: landscape complexity (percent seminatural habitat in the most 692 predictive radius); D: sampling date (1-3); T: exclusion treatment (6 levels of natural enemy 693 exclusion)

697 Fig. 1. Effects of landscape complexity and natural enemy exclusion on average daily aphid population growth across three sampling dates (1-3). Average daily population growth is the log-

699 ratio of aphid density on sampling dates 1 to 3 and of initial aphid density, divided by the

700 number of days since the start of the experiment (date 1: after 10 days, date 2: after 20 days, date

701 3: after 30 days). The top half of the figure shows mean growth \pm s.e.m. per exclusion treatment

702 and sampling date. The lower half shows variation of predicted values across the gradient in 703 landscape complexity, measured by percent seminatural habitat in a $700 \mathrm{~m}$ radius around fields.

704 Data points per treatment and date are provided in Fig. S3. See Table S3 for multiple slope 705 comparisons. Different letters indicate significant differences between treatments. In the legend, 
706

707

708

709

710

711 Fig. 2. Effects of landscape complexity and natural enemy exclusion on (A) aphid parasitism rate

crossed-out symbols indicate exclusion of natural enemy functional guilds. Treatments remain accessible to non-excluded guilds. Guilds of natural enemies include flying insects (parasitoids, syrphid flies and predatory wasps; wasp symbol), ground-dwellers (carabid beetles, staphylinids and spiders; beetle symbol) and birds (and other vertebrates larger than $1.5 \mathrm{~cm}$; bird symbol).

712 and (B) syrphid fraction at three sampling dates (dates 1-3; 10 day intervals). The top half of

713 each figure shows mean values \pm s.e.m. per exclusion treatment and sampling date. The lower

714 half shows variation of predicted values across the gradient in landscape complexity, measured

715 by percent seminatural habitat in a $200 \mathrm{~m}$ and $900 \mathrm{~m}$ radius around fields for parasitism rates and

716 syrphid fractions, respectively. Data points per treatment and date are provided in Figs S4, S5.

717 See Table S3 for slope multiple comparisons. Different letters indicate significant differences

718 between treatments. Detailed legend description is provided in Fig. 1.

719

720

721

Fig. 3. Relationship between aphid population growth and (A) parasitism rate $(\mathrm{n}=601),(\mathrm{B})$ syrphid fraction ( $\mathrm{n}=588)$ and $(\mathrm{C})$ between final cabbage biomass and syrphid fraction (mean of

722 sampling dates; $\mathrm{n}=84)$.

723

724 


\section{Table $\mathbf{1}_{\text {(on next page) }}$}

Results of model selection relating landscape complexity and enemy exclusion to response variables.

Model lists show the $95 \%$ best models at the most predictive scale for each response variable. w: AIC weight compared to all possible models, w95\%: AIC weight within the 95\% model confidence set. The sum of weights for each term is the sum of AIC weights of all models selecting it and represents the probability of being present in the $95 \%$ model confidence set. Selected explanatory variables are M: management of the nearest surrounding field (organic / conventional); L: landscape complexity (percent seminatural habitat in the most predictive radius); D: sampling date (1-3); T: exclusion treatment (6 levels of natural enemy exclusion) 


\begin{tabular}{|c|c|c|c|c|c|c|c|c|c|c|c|c|c|c|c|}
\hline \multirow{2}{*}{$\begin{array}{l}\text { Response } \\
\text { aphid population } \\
\text { growth }\end{array}$} & \multirow{2}{*}{$\frac{\mathbf{n}^{\circ}}{\mathrm{M} 1}$} & \multicolumn{9}{|c|}{ Model specification } & \multirow{2}{*}{$\frac{\mathbf{d f}}{52}$} & \multirow{2}{*}{$\begin{array}{l}\text { AICc } \\
-3480\end{array}$} & \multirow{2}{*}{$\frac{\Delta \mathbf{A I C}}{0}$} & \multirow{2}{*}{$\frac{\mathbf{w}}{0.94}$} & \multirow{2}{*}{$\begin{array}{l}\begin{array}{l}\text { w } \\
\mathbf{9 5 \%}\end{array} \\
1\end{array}$} \\
\hline & & $M+$ & $L+$ & $D+$ & $T+$ & $\mathrm{M}: \mathrm{D}+$ & $L: D+$ & $\mathrm{T}: \mathrm{D}+$ & $\mathrm{L}: \mathrm{T}+$ & L:T:D & & & & & \\
\hline \multicolumn{2}{|c|}{ Sum of weights } & 1 & 1 & 1 & 1 & 1 & 1 & 1 & 1 & 1 & & & & 0.94 & 1 \\
\hline \multirow[t]{6}{*}{ parasitism rate $\sim$} & M2 & $M+$ & $L+$ & $D+$ & $\mathrm{T}+$ & M:D + & L:D + & & $\mathrm{L}: \mathrm{T}$ & & 23 & 1486.8 & 0.00 & 0.71 & 0.75 \\
\hline & M3 & $M+$ & $\mathrm{L}+$ & $D+$ & $\mathrm{T}+$ & M:D + & & & $\mathrm{L}: \mathrm{T}$ & & 21 & 1491.6 & 4.87 & 0.06 & 0.07 \\
\hline & M4 & & $\mathrm{L}+$ & $D+$ & $T+$ & & $L: D+$ & & $\mathrm{L}: \mathrm{T}$ & & 20 & 1491.8 & 5.03 & 0.06 & 0.06 \\
\hline & M5 & $M+$ & $L+$ & $D+$ & $T+$ & & $L: D+$ & & $\mathrm{L}: \mathrm{T}$ & & 21 & 1492.1 & 5.34 & 0.05 & 0.05 \\
\hline & M6 & $M+$ & $\mathrm{L}+$ & $D+$ & $\mathrm{T}+$ & $M: D+$ & $L: D+$ & $\mathrm{T}: \mathrm{D}+$ & $\mathrm{L}: \mathrm{T}$ & & 33 & 1492.8 & 6.03 & 0.04 & 0.04 \\
\hline & M7 & $M+$ & $\mathrm{L}+$ & $D+$ & $\mathrm{T}+$ & $M: D+$ & $L: D$ & & & & 18 & 1493 & 6.26 & 0.03 & 0.03 \\
\hline \multicolumn{2}{|c|}{ Sum of weights } & 0.94 & 1 & 1 & 1 & 0.89 & 0.14 & 0.04 & 0.97 & & & & & 0.95 & 1.00 \\
\hline \multirow[t]{11}{*}{ syrphid fraction } & M8 & $M+$ & $\mathrm{L}+$ & $D+$ & $\mathrm{T}+$ & & & & $\mathrm{L}: \mathrm{T}$ & & 19 & 1229.6 & 0.00 & 0.23 & 0.25 \\
\hline & M9 & & $\mathrm{L}+$ & $D+$ & $\mathrm{T}+$ & & & & $\mathrm{L}: \mathrm{T}$ & & 18 & 1230 & 0.35 & 0.20 & 0.21 \\
\hline & M10 & $\mathrm{M}+$ & $L+$ & $D+$ & $\mathrm{T}+$ & M:D + & & & $\mathrm{L}: \mathrm{T}$ & & 21 & 1230.2 & 0.58 & 0.18 & 0.19 \\
\hline & M11 & $M+$ & $\mathrm{L}+$ & $D+$ & $T+$ & $\mathrm{M}: \mathrm{D}+$ & $L: D+$ & & $\mathrm{L}: \mathrm{T}$ & & 23 & 1232.1 & 2.47 & 0.07 & 0.07 \\
\hline & M12 & $M+$ & & $D+$ & $T+$ & $M: D$ & & & & & 15 & 1232.4 & 2.78 & 0.06 & 0.06 \\
\hline & M13 & $M+$ & & $D+$ & $\mathrm{T}+$ & & & & & & 13 & 1232.5 & 2.82 & 0.06 & 0.06 \\
\hline & M14 & $M+$ & $\mathrm{L}+$ & $D+$ & $T+$ & & $L: D+$ & & $\mathrm{L}: \mathrm{T}$ & & 21 & 1233 & 3.36 & 0.04 & 0.05 \\
\hline & M15 & & $\mathrm{L}+$ & $D+$ & $\mathrm{T}+$ & & $L: D+$ & & $\mathrm{L}: \mathrm{T}$ & & 20 & 1233.2 & 3.58 & 0.04 & 0.04 \\
\hline & M16 & & & $D+$ & $\mathrm{T}+$ & & & & & & 12 & 1233.3 & 3.65 & 0.04 & 0.04 \\
\hline & M17 & $M+$ & $\mathrm{L}+$ & $D+$ & $\mathrm{T}+$ & M:D & & & & & 16 & 1234.3 & 4.67 & 0.02 & 0.02 \\
\hline & ights & 0.71 & 0.84 & 1 & 1 & 0.35 & 0.16 & & 0.81 & & & & & 0.93 & 1.00 \\
\hline
\end{tabular}




\section{Figure 1 (on next page)}

Effects of landscape complexity and natural enemy exclusion on average daily aphid population growth across three sampling dates (1-3).

Average daily population growth is the log-ratio of aphid density on sampling dates 1 to 3 and of initial aphid density, divided by the number of days since the start of the experiment (date 1: after 10 days, date 2: after 20 days, date 3: after 30 days). The top half of the figure shows mean growth \pm s.e.m. per exclusion treatment and sampling date. The lower half shows variation of predicted values across the gradient in landscape complexity, measured by percent seminatural habitat in a $700 \mathrm{~m}$ radius around fields. Data points per treatment and date are provided in Fig. S3. See Table S3 for multiple slope comparisons. Different letters indicate significant differences between treatments. In the legend, crossed-out symbols indicate exclusion of natural enemy functional guilds. Treatments remain accessible to non-excluded guilds. Guilds of natural enemies include flying insects (parasitoids, syrphid flies and predatory wasps; wasp symbol), ground-dwellers (carabid beetles, staphylinids and spiders; beetle symbol) and birds (and other vertebrates larger than $1.5 \mathrm{~cm}$; bird symbol). 


\section{PeerJReviewing Manuscript}

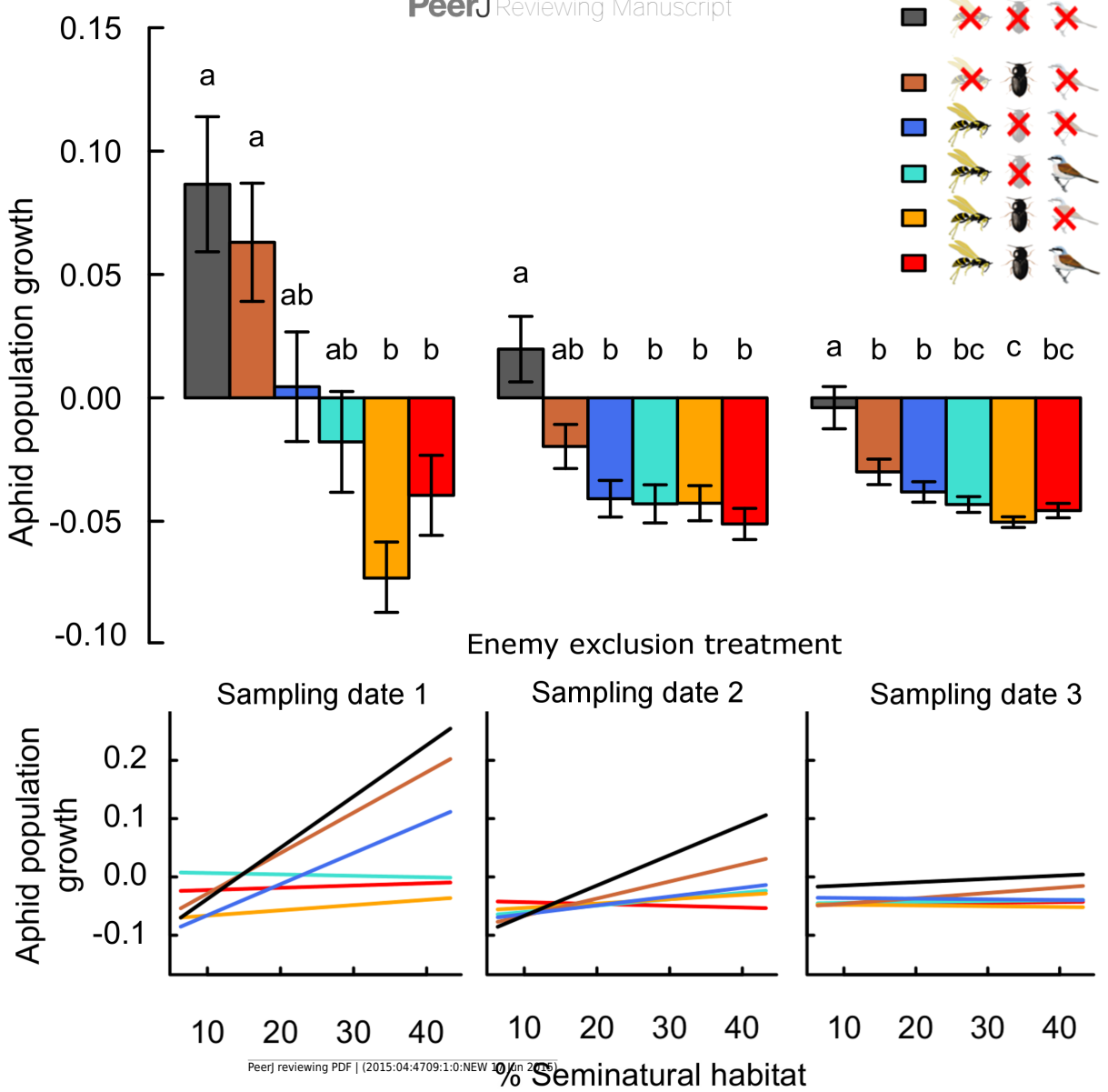




\section{Figure 2 (on next page)}

Effects of landscape complexity and natural enemy exclusion on (A) aphid parasitism rate and (B) syrphid fraction at three sampling dates (dates 1-3; 10 day intervals).

The top half of each figure shows mean values \pm s.e.m. per exclusion treatment and sampling date. The lower half shows variation of predicted values across the gradient in landscape complexity, measured by percent seminatural habitat in a $200 \mathrm{~m}$ and $900 \mathrm{~m}$ radius around fields for parasitism rates and syrphid fractions, respectively. Data points per treatment and date are provided in Figs S4, S5. See Table S3 for slope multiple comparisons. Different letters indicate significant differences between treatments. Detailed legend description is provided in Fig. 1. 
Figure 3 (on next page)

Relationship between aphid population growth and (A) parasitism rate $(n=601)$, (B) syrphid fraction $(n=588)$ and $(C)$ between final cabbage biomass and syrphid fraction (mean of sampling dates; $n=84$ ). 
